\title{
Transmylohyoid Orotracheal Intubation: An Expedient Method of Airway Management in Multiple Facial Fractures
}

\author{
${ }^{1}$ Rajesh Kshirsagar, ${ }^{2}$ Vrushika H Mahajan, ${ }^{3}$ Vishal Tapadiya, ${ }^{4}$ Sudhir Pawar
}

\begin{abstract}
Aim: The purpose of this article is to reiterate the importance of transmylohyoid orotracheal intubation in panfacial fractures that require maxillomandibular fixation (MMF) followed by assessment of occlusion and the need to avoid tracheostomy. Maxillofacial injuries are very common following road traffic accidents. Many times, injured patients present with panfacial fractures and need intraoperative MMF to attain dental occlusion, setting unique anesthetic challenges. A choice often has to be made between orotracheal and nasotracheal when surgical access to the nasal or oral cavity is necessary. A 28-year-old obese male patient was scheduled for the management of panfacial fracture sustained in a motorcycle accident. Transmylohyoid orotracheal intubation was performed due to the need for MMF. Transmylohyoid orotracheal intubation is a simple, secure, and effective procedure for operative airway control in major panfacial traumas.
\end{abstract}

Clinical significance: Transmylohyoid orotracheal intubation should be chosen over tracheostomy in panfacial fractures that manifest as MMF.

Keywords: Airway management, Maxillofacial trauma, Transmylohyoid orotracheal intubation.

How to cite this article: Kshirsagar R, Mahajan VH, Tapadiya V, Pawar S. Transmylohyoid Orotracheal Intubation: An Expedient Method of Airway Management in Multiple Facial Fractures. World J Dent 2017;8(5):422-424.

Source of support: Nil

Conflict of interest: None

\section{INTRODUCTION}

The maxillofacial surgeon and the anesthesiologists share the maxillofacial region for their work. ${ }^{1}$ Thus, working remains a challenge for both specialists in patients with panfacial fractures.

An orotracheal intubation precludes the assessment of dental occlusion as well as the intraoperative use of MMF. ${ }^{1}$ Anesthesiologists may be reluctant to use nasotracheal intubation in cases of naso-orbito-ethmoid (NOE) and skull base fractures. If performed, it may interfere

\footnotetext{
${ }^{1-4}$ Department of Oral and Maxillofacial Surgery, Bharati Vidyapeeth Deemed University Dental College and Hospital Pune, Maharashtra, India

Corresponding Author: Vrushika H Mahajan, Department of Oral and Maxillofacial Surgery, Bharati Vidyapeeth Deemed University Dental College and Hospital, Pune, Maharashtra India, e-mail: mahajanvrushika@gmail.com
}

with the treatment of nasal or midface fractures. Tracheostomy remains a substitute for establishing a surgical airway, allowing accessibility and achievement of proper dental occlusion in such cases. ${ }^{2}$ However, tracheostomy requires surgical expertise, diligent postoperative care, and is known to cause nonesthetic visible scarring. The transmylohyoid orotracheal intubation is an excellent alternative to maintain the airway during maxillofacial surgeries, with access to all fracture sites, intraoperative assessment of occlusion, and use of MMF.

The medical records of 40 patients presenting panfacial trauma, who underwent transmylohyoid intubation from 2011 to 2015, were reviewed. In our retrospective evaluation for the safety and efficacy, it was evident that transmylohyoid intubation was completed successfully in all patients. The average time required for intubation was 7 minutes. There was no incident of accidental extubation. No accidental extubations or tube injuries occurred. Ten cases of superficial infection of the extraoral wound were noted. Scars were imperceptible after 3 months.

\section{CASE REPORT}

A 28-year-old obese male patient was scheduled for management of panfacial fracture sustained in a motorcycle accident. The patient had reduced mouth opening $(15 \mathrm{~mm})$ and deranged occlusion. Nasal bone fractures did not permit the use of nasotracheal intubation, and the need for intraoperative MMF ruled out orotracheal intubation. The transmylohyoid intubation appeared to be the most suitable option.

After a normal orotracheal intubation with a flexometallic endotracheal tube (ETT), a paramedian skin incision $(1.5-2 \mathrm{~cm})$ was made. This incision was parallel to the lower border of the mandible and placed at a fingerbreadth on the medial aspect of the inferior border of the mandible. The platysma and deep cervical fascia were bluntly dissected with the artery forceps always close to the lingual cortex of the mandible. The mylohyoid muscle was traversed to reach the sublingual space. A mucosal incision was made parallel with the gingival margin. It is advisable to pass a finger through the skin incision to appear intraorally to ensure the patency of the tract before attempting to pass the tube through the floor of the mouth. Then, the cuff was deflated and taken out through the incision. Then, ETT was pulled out using an 


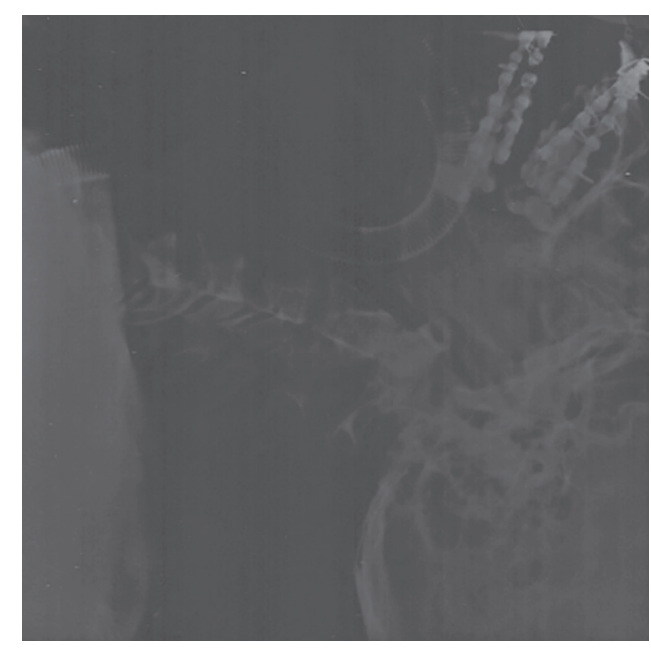

Fig. 1: Intraoperative radiograph showing the transmylohyoid orotracheal intubation

hemostat. The cuff was inflated and airway was secured. The ETT was secured with stay sutures. Intraoperative radiograph was taken to assess the path of ETT (Fig. 1).

Dental occlusion was restored by MMF followed by open reduction and internal fixation of the fractures. At the end, stay sutures were removed, and the ETT was brought back into the oral cavity. The skin wound was sutured while the intraoral wound was left to heal with secondary intention. The patient was extubated at the conclusion of the procedure.

\section{DISCUSSION}

Airway management for patients afflicted with panfacial fractures is a challenge for the anesthetic team. In addition, the maxillofacial surgeon requires intraoperative MMF to achieve satisfactory surgical outcomes. This necessitates modification of the standard anesthesia techniques.

Orotracheal intubation despite a good mouth opening is not suitable in patients with midfacial and panfacial fractures as the surgeon cannot assess the occlusion.

Nasotracheal intubation is often contraindicated in the presence of comminuted midface fractures and fracture of base of the skull as it may lead to cranial intubation, displacement of comminuted fracture fragments, epistaxis, trauma to the pharynx, otitis media, sepsis, and inability to pass a tube through the nasal passage. ${ }^{3}$ Fiberoptic-guided nasotracheal intubation requires advanced expensive equipment and technical expertise, thus limiting its use at primary health care center. ${ }^{4}$ In these conditions, tracheostomy may be indicated, but is known to be associated with hemorrhage, surgical emphysema, wound site infection, recurrent laryngeal nerve injury, tracheal stenosis, poor scar esthetics, fistula, and death. ${ }^{5}$ Retromolar intubation technique ${ }^{6}$ can be an alternative in such cases, but its demerits include more time for retromolar region bone removal, chances of lingual nerve injury, and tube compression.

Hernández Altemir $\mathrm{F}^{7}$ documented the submental route for endotracheal intubation to avoid tracheotomy in maxillofacial trauma where short-term MMF is required.

However, this technique remains underutilized by the surgical and anesthetic fraternities. Gadre and Kushte $^{8}$ suggested that the term "transmylohyoid orotracheal intubation" as the path of exit of ETT is across the mylohyoid muscle and not restricted to the submental triangle. Gadre and Waknis ${ }^{9}$ concluded that ETT can pass anywhere between the mandibular first molar regions on either side without being restricted to the submental triangle.

Arya et al ${ }^{10}$ modified the technique to retrograde submental intubation by pharyngeal loop technique where the initial step of orotracheal intubation is not possible because of restricted mouth opening.

Transmylohyoid intubation is indicated in cases of panfacial fractures, NOE complex fractures, skull base fractures, transmaxillary skull base tumors, pathologies of maxillofacial region, congenital deformities, elective facial osteotomy, and requirement of short-term airway maintenance ( $>7$ days).

Few contraindications include existing infection at the site of incision, bleeding diathesis, disrupted laryngotracheal anatomy, and requirement of prolonged maintenance of airway ( $>7$ days).

Advantages of transmylohyoid intubation include the following: It aids in MMF and occlusion assessment, allows downward retraction of maxilla, increases surgical exposure for transmaxillary skull base surgeries, provides supplementary working area around the operating field, is a quick method of intubation, requires no specialized equipment or technical expertise, can be easily used in obese patients, helps in early extubation of the patient after operation, and avoids postoperative tracheostomy tube care and complications of tracheostomy.

Complications of transmylohyoid intubation include infection in the floor of the mouth and at the site of skin incision, damage to the tube apparatus, risk of orocutaneous fistulae, accidental extubation, excessive bronchial flexion, venous bleeding, dislodgement of throat pack sticker, mucocele, transient lingual nerve paresthesia, damage to the important structures in the floor of the mouth, and hypertrophic scar.

\section{CONCLUSION}

Where traditional airway securing methods are contraindicated, the communication between the surgeon and the anesthetist is paramount. The surgeon should 
suggest surgical airway techniques to the anesthetist. At our institute, we exercised transmylohyoid intubation in 40 panfacial trauma patients requiring MMF. It revealed the technique to be extremely useful as it is time-saving and less technique sensitive requiring no additional instrumentation with minimal complications.

\section{CLINICAL SIGNIFICANCE}

Transmylohyoid orotracheal intubation should be chosen over tracheostomy in panfacial fractures, which manifest as MMF.

\section{REFERENCES}

1. Biglioli F, Mortini P, Goisis M, Bardazzi A, Boari N. Submental orotracheal intubation: an alternative to tracheotomy in transfacial cranial base surgery. Skull Base 2003 Nov;13(4):189-195.

2. Caron G, Paquin R, Lessard MR, Trépanier CA, Landry PE. Submental endotracheal intubation: an alternative to tracheotomy in patients with midfacial and panfacial fractures. J Trauma 2000 Feb;48(2):235-240.
3. Muzzi DA, Losasso TJ, Cucchiara RF. Complication from a nasopharyngeal airway in a patient with a basilar skull fracture. Anesthesiology 1991 Feb;74(2):366-368.

4. Kumar V, Singh AK, Kumar P, Shenoy YR, Verma AK, Borole AJ, Prasad V. Blast injury face: an exemplified review of management. Natl J Maxillofac Surg 2013 Jan-Jun;4(1):33-39.

5. Johnson TR. Submental intubation versus tracheostomy. $\mathrm{Br}$ J Anaesth 2002 Aug;89(2):344-345.

6. Martinez-Lage JL, Eslava JM, Cebrecos AI, Marcos O. Retromolar intubation. J Oral Maxillofac Surg 1998 Mar;56(3): 302-306.

7. Hernández Altemir F. The submental route for endotracheal intubation. A new technique. J Maxillofac Surg 1986 Feb;14(1):64-65.

8. Gadre KS, Kushte D. Transmylohyoid oroendotracheal intubation: a novel method. J Craniofac Surg 1992 Jul;3(1):39-40.

9. Gadre KS, Waknis PP. Transmylohyoid/Submental intubation: review, analysis, and refinements. J Craniofac Surg 2010 Mar;21(2):516-519.

10. Arya VK, Kumar A, Makkar SS, Sharma RK. Retrograde submental intubation by pharyngeal loop technique in a patient with faciomaxillary trauma and restricted mouth opening. Anesth Analg 2005 Feb;100(2):534-537. 